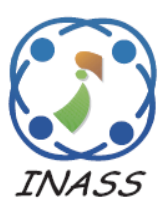

\title{
An Improved Robust Fractionalized PID Controller for a Class of Fractional-Order Systems with Measurement Noise
}

\author{
Yassine Bensafia ${ }^{1}$ \\ Khatir Khettab $^{2 *}$ \\ Abdelhakim Idir ${ }^{3}$ \\ ${ }^{1}$ Department of Electrical Engineering, Bouira University, 10000, Algeria. \\ ${ }^{2}$ Department of Electrical Engineering, University Mohamed Boudiaf of M'sila, 28000, Algeria \\ ${ }^{3}$ Department of Electrical Engineering, University M'Hamed Bougara of Boumerdes, 35000, Algeria. \\ * Corresponding author's Email: zoubirhh@yahoo.fr
}

\begin{abstract}
Recently, many research works have focused on fractional order control (FOC) and fractional systems. It has proven to be a good mean for improving the plant dynamics with respect to response time and disturbance rejection. In this paper we propose a new approach for robust control by fractionalizing an integer order integrator in the classical PID control scheme and we use the Sub-optimal Approximation of fractional order transfer function to design the parameters of PID controller, after that we study the performance analysis of fractionalized PID controller over integer order PID controller. The implementation of the fractionalized terms is realized by mean of well-established numerical approximation methods. Illustrative simulation examples show that the disturbance rejection is improved by $50 \%$. This approach can also be generalized to a wide range of control methods.
\end{abstract}

Keywords: Fractional order control, PID controller, Robust control, Sub-optimal approximation, Noises.

\section{Introduction}

Even if the great popularity of fractional calculus is very recent mainly regarding its application in science and engineering, its history goes 300 years back.

Particularly, control theory and applications is one of the major fields of application of fractional order systems, with a quickly growing quantity of theoretical and experimental research production [1].

The reason for this success is due to the advantageous properties of fractional order control (FOC) systems and their interesting ability to improve the process robustness against disturbances and noises. A good confirmation is the fact that the first FOC scheme ever proposed in the literature, the so-called "Commande Robuste d'Ordre Non Entier" (CRONE) controller [2], deals with robust control. It uses the constant phase property of the ideal Bode's transfer function $1 / \mathrm{s}^{\alpha}$ to obtain a robust feedback control against gain variations.

Another factor is that using fractional order filters in feedback control applications, presents a certain advantageous action on the system dynamical behavior. This is due to the hereditary property of fractional order operators [3] offering an interesting robustness improvement versus external noises [4-6].

A great research effort is focused nowadays on the design and analysis of new robust fractional order controllers on the basis of the CRONE control approach [8]. Another pioneering contribution was the proposition of combining the classical wellestablished PID controller with fractional order differentiation, introduced by Podlubny [7]. He developed a generalization of this controller called the PID controller, involving an integration action of order $\lambda$ and a differentiation action of order $\mu$. The problem of tuning and performance improvement of fractional order PID controllers was the new challenge towards practical usage of this generalized PID controller in industrial processes [8-10]. Consequently, the number of robust fractional order control applications is growing exponentially touching various physical processes as can be found in the fractional control literature [7, 11-13]. 
Robustness is one of the main advantages of Fractional Order Control (FOC). In the other hand, it has been proven that the use of fractional order systems which are long memory processes in feedback control systems, presents a certain benefit action on the system dynamical behavior and a good robustness effect against noises and perturbations [14-16].

Many research works have then focused on new robust fractional order controllers design mainly based on the CRONE strategy $[8,9]$.

In [16], the authors propose a new FOC design based on a robust Fractionalized Adaptive PI control tuning method.

However, this also implies that the tuning of the controller can be much more complex. In order to address this problem, different methods for the design of a FOPID controller have been proposed in the literature $[3,12]$.

The concept of FOPID controllers was proposed by Podlubny in [22]. He also demonstrated the better response of this type of controller, in comparison with the classical PID controller, when used for the control of fractional order systems [23].

There are other papers published in the recent years where the tuning of PID controller via PSO such as [28], Lyapunov-Gain-Scheduled [29] and Harmony Search Algorithms [30] was investigated.

The main contribution of this work is the use the fractionalized PID controller approach to reduce noise effect by introducing fractional order filters in the classical feedback control loop PID controller without changing the overall equivalent closed loop transfer function.

This paper is structured as follows: Section 2 is a fundamental of fractional calculus. Section 3 presents numerical algorithm for Sub-optimal Rational Approximations, and the fractionalized PID controller and simulation results are given is given in section 4 and 5. Finally, the conclusion with future work is presented in section 6 .

\section{Fundamentals of fractional calculus}

\subsection{Definition of fractional calculus}

Fractional calculus is a subdivision of calculus theory which generalizes the derivative or integral of a function to non-integer order $[5,20]$. The number of applications where fractional calculus has been used grows rapidly mainly for the reason that these mathematical phenomena allow to describe a real object more accurately than the classical methods. Approximation methods of fractional derivative and integral to rational functions permitted to use very easily fractional order systems in wide areas of applications such as control theory $[5,8,12]$, economical systems [20], renewable energy, fractional chaotic systems $[21], \ldots$ etc.

The generalized fundamental operator which includes the differentiation and integration is given as:

$$
a D_{t}^{q}= \begin{cases}\frac{d^{q}}{d t^{q}} & , R(q)>0 \\ 1 & , R(q)=0 \\ \int_{a}^{t}(d \tau)^{-q} & , R(q)<0\end{cases}
$$

Where,

$a$ : Lower limit of integration

$t$ : Upper limit of integration

$q$ : Order of fractional differentiation or integration

$q$ with negative value indicates integration while $q$ with positive value indicates differentiation. The theory of fractional-order derivative was developed mainly in the 19th century. There are several definitions of fractional order derivative. Two important and widely applied definitions are Grunwald-Letnikov definition is perhaps the best known due to its most suitability for the realization of discrete control algorithms [1, 12]. The Grunwald-Letnikov definition is expresses as [21, 24-27]:

$$
D^{\alpha} f(t)=\lim _{h \rightarrow 0} h^{-\alpha} \sum_{j=0}^{k}(-1)^{j}\left(\begin{array}{l}
\alpha \\
j
\end{array}\right) f(k h-j h)
$$

Where the coefficients are evaluated from:

$\omega_{j}^{(\alpha)}=\left(\begin{array}{l}\alpha \\ j\end{array}\right)=\frac{(\alpha+1)}{(j+1)(\alpha-j+1)}$

and $h$ is the step time .

The Riemann-Liouville definition is expresses as:

$$
f(t)=\frac{d^{n}}{d t^{n}} \int_{O}^{t} \frac{f(\tau)}{(t-\tau)^{\alpha-n+1}} d \tau
$$

For a wide class of functions which appear in real physical and engineering applications, the RiemannLiouville and the Grunwald-Letnikov definitions are equivalent [2, 25-27].

\subsection{Fractional order systems}

Feedback control system is one of the major areas where the concept of fractional calculus is being applied to obtain an efficient system and also longevity and freedom. To the control engineer to compensate any shifts in the transfer function due to 
parametric spreads, aging etc. a system is efficient if the controller is of the similar order to that of a plant being controlled. In reality the systems are of fractional order and therefore to have a fractional order controller will be efficient $[3,12]$. The transfer function of a fractional order system is given as:

$$
G(s)=\frac{b_{m} s^{\beta_{m}}+b_{m-1} s^{\beta_{m-1}+\cdots+b_{1} s^{\beta_{1}}+b_{0}}}{a_{n} s^{\alpha_{n}+a_{n-1} s^{\alpha_{n-1+\cdots}}}}
$$

where $a_{i}$ and $b_{j}$ are real numbers such that

$$
\left\{\begin{array}{l}
0 \leq \alpha_{0} \leq \alpha_{1} \leq \cdots \leq \alpha_{n} \\
0 \leq \beta_{0} \leq \beta_{1} \leq \cdots \leq \beta_{m}
\end{array}\right.
$$

and $s$ is the Laplace operator.

\section{Rational approximations to fractional integrators and differentiators: Outstaloup's method}

The approximation of Oustaloup a generalized derivator, differential action which covers the frequency space, based on a recursive distribution of an infinite number of zeros and negative real poles (to ensure phase behavior minimum). As part of a realist synthesis (practice) based on a finite number of zeros and poles, it should reduce the differential behavior of a generalized bounded frequency range, chosen according to the needs of the application [1719].

The method is based on the function approximation from:

$$
H(s)=S^{\alpha} \quad, \alpha \in R^{+}
$$

By a rational function [16]:

$$
G_{f}(s)=K \prod_{k=1}^{N} \frac{s+w_{k}^{\prime}}{s+w_{k}}
$$

Where the poles, zeros, and gain are evaluated from:

$$
\begin{aligned}
& w_{k}^{\prime}=w_{b} \cdot w_{u}^{\frac{2 k-1-\gamma}{N}} \\
& w_{k}=w_{b} \cdot w_{u}^{(2 k-1+\gamma) / N}, K=w_{h}^{\gamma}
\end{aligned}
$$

Where $w_{u}$ is the unity frequencies gain and the central frequency of a band of frequencies distributed geometrically. Let $w_{u}=\sqrt{w_{h} w_{b}}$, where $w_{h}$ and $w_{b}$ are respectively the upper and lower frequencies. $\gamma$ is the order of derivative, and $N$ is the order of the filter.

\section{A numerical algorithm for sub-optimal rational approximations}

Our target now is to find an approximate integerorder model with a relative low order, possibly with a time delay in the following form:

$$
G_{r / m, \tau}(s)=\frac{\beta_{1} s^{r}+\cdots+\beta_{r} s+\beta_{r+1}}{s^{m}+\alpha_{1} s^{m-1}+\cdots+\alpha_{m-1} s+\alpha_{m}} e^{-\tau s}
$$

An objective function for minimizing the $\mathrm{H}_{2}$-norm of the reduction error signal $e(t)$ can be defined as:

$$
J=\min _{\theta}\left\|\widehat{G}(s)-G_{r / m, \tau}(s)\right\|_{2}
$$

where $\theta$ is the set of parameters to be optimized such that

$$
\theta=\left[\beta_{1}, \ldots, \beta_{r}, \alpha_{1}, \ldots, \alpha_{m}, \tau\right]
$$

For an easy evaluation of the criterion $\mathrm{J}$, the delayed term in the reduced order model $G_{r / m, \tau}(s)$ can be further approximated by a rational function $G_{r / m}(s)$ using the Padé approximation technique [12]. Thus, the revised criterion can then be defined by

$$
J=\min _{\theta}\left\|\hat{G}(s)-\widehat{G}_{r / m}(s)\right\|_{2}
$$

and the $\mathrm{H}_{2}$ norm computation can be evaluated recursively using the algorithm in $[1,12,13]$.

Suppose that for a stable transfer function type

$$
E(s)=\widehat{G}(s)-\widehat{G}_{r / m}(s)=B(s) / A(s)
$$

the polynomials $A_{k}(s)$ and $B_{k}(s)$ can be defined such that

$$
\begin{aligned}
& A_{k}(s)=a_{0}^{k}+a_{1}^{k} s+\cdots+a_{k}^{k} s^{k}, \quad B_{k}(s)=b_{0}^{k}+ \\
& b_{1}^{k} s+\cdots+b_{k-1}^{k} s^{k-1}
\end{aligned}
$$

The values of $a_{i}^{k-1}$ and $b_{i}^{k-1}$ can be evaluated recursively from

$$
a_{i}^{k-1}=\left\{\begin{array}{c}
a_{i+1}^{k} \quad \begin{array}{c}
\text { i iven } \\
a_{i+1}^{k}-\alpha_{k} a_{i+2}^{k}, \quad \text { i odd } \\
i=0, \ldots, k-1
\end{array}
\end{array}\right.
$$

and

$$
b_{i}^{k-1}=\left\{\begin{array}{c}
b_{i+1}^{k} \quad \text { i iven } \\
b_{i+1}^{k}-\beta_{k} a_{i+2}^{k}, \quad \text { i odd } \\
i=0, \ldots, k-1
\end{array}\right.
$$

Where $\alpha_{k}=a_{0}^{k} / a_{1}^{k}$ and $\beta_{k}=b_{1}^{k} / a_{1}^{k}$ 
The H2-norm of the approximate reduction error signal $\hat{e}(\mathrm{t})$ can be evaluated from:

$$
J=\sum_{k=1}^{n} \frac{\beta_{k}^{2}}{2 \alpha_{k}}=\sum_{k=1}^{n} \frac{\left(b_{1}^{k}\right)^{2}}{2 a_{0}^{k} a_{1}^{k}}
$$

The sub-optimal H2-norm reduced order model for the original high-order fractional-order model can be obtained using the following procedure [2]:

1. Select an initial reduced model $\hat{G}_{r / m}^{0}(s)$

2. Evaluate an error $\left\|\hat{G}(s)-\widehat{G}_{r / m}^{0}(s)\right\|_{2}$ from (9).

3. Use an optimization algorithm (for instance, Powell's algorithm]) to iterate one step for a better estimated model $\hat{G}_{r / m}^{1}(s)$.

4. Set $\hat{G}_{r / m}^{0}(s) \longleftarrow \widehat{G}_{r / m}^{1}(s)$, go to Step 2 until an optimal reduced model $\hat{G}_{r / m}^{*}(s)$ is obtained.

5. Extract the delay from $\hat{G}_{r / m}^{*}(s)$, if any.

\section{Fractionalized-order PID controller}

The feedback control loop with an integer order controller is shown in Fig. 1, where $\mathrm{U}_{\mathrm{R}}(\mathrm{s})$ is an input signal, $\mathrm{E}(\mathrm{s})$ is an error signal, $\mathrm{C}(\mathrm{s})$ is a controller transfer function, $\mathrm{G}(\mathrm{s})$ is a system or plant transfer function, $\mathrm{Y}(\mathrm{s})$ is an output signal, and $\mathrm{U}(\mathrm{s})$ is a control signal

The integer-order PID controller to be designed is in the following form:

$$
C(s)=K_{p}\left(1+\frac{1}{T_{i} s}+T_{d} s\right)
$$

The PID control scheme is modified here to get more robustness against noise and perturbation. The new PID control law is obtained by using the fractionalization of a control system element [1, 2, 16], the integral operator $1 / \mathrm{s}$ is fractionalized as represented in Fig. 2, that is,

$$
\frac{1}{S}=\frac{1}{S^{\alpha}} \frac{1}{S^{(1-\alpha)}}
$$

where $\alpha$ is a real number such that $0<\alpha<1$.

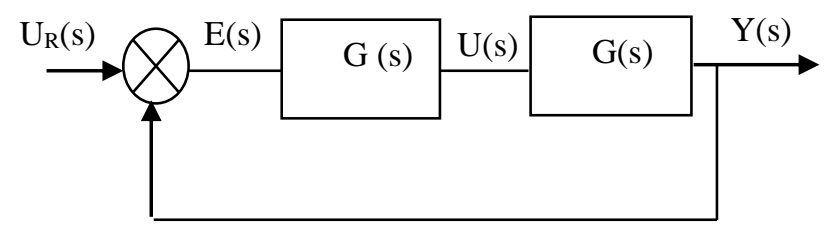

Figure.1 Feedback control loop with an integer order controller

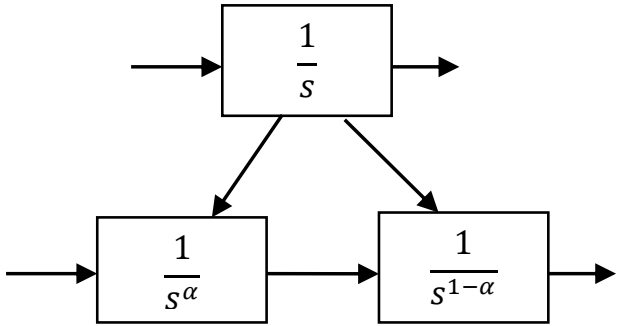

Figure. 2 Fractionalization of integral operator.



Figure.3 Feedback control loop with a fractionalized order controller

Our proposed methodology does not change the original global stable control scheme, but may improve its robustness against external noise and perturbation, by taking benefit of the interesting properties of fractional order systems. This new idea even though simple and easy to implement, opens a new manoeuvring margin to the design engineers dealing with plants in realistic industrial conditions.

The feedback control loop with a fractionalized order controller is shown in Fig. 3, where $C_{f}(s)$ is a fractionalized controller transfer function.

The fractionalized of the integer-order PID controller to be designed is in the following form:

$$
\begin{aligned}
C_{f}(s) & =K_{p}\left(1+\frac{1}{T_{i} s}+T_{d} s\right) \\
& =\frac{1}{s}\left(\frac{\left(k_{p} T_{d} s^{2}+k_{p} T_{i} s+k_{p}\right.}{T_{i}}\right) \\
& =\frac{1}{s^{\alpha}} \frac{1}{S^{(1-\alpha)}}\left(\frac{\left(k_{p} T_{d} s^{2}+k_{p} T_{i} s+k_{p}\right.}{T_{i}}\right)
\end{aligned}
$$

Were, $0<\alpha<1$.

\section{Simulation results and discussion}

Let us consider the following FO-LTI plant model:

$$
G(s)=\frac{1}{s^{2.3}+3.2 s^{1.4}+2.4 s^{0.9}+1}
$$

Let us first approximate it with Oustaloup's method and then fit it with a fixed model structure known as first-order lag plus dead time (FOLPD) model, where

$$
G_{r}=\frac{K}{T s+1} e^{-L s}
$$




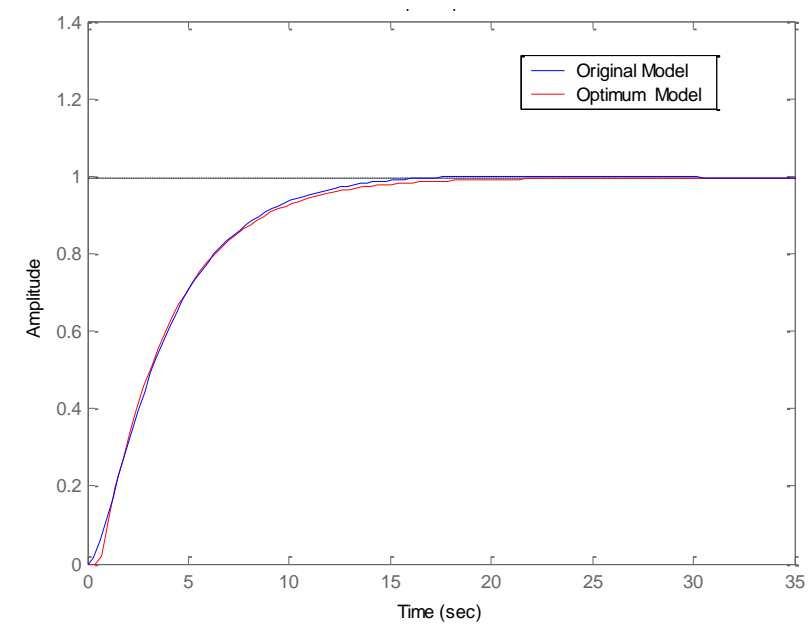

Figure.4 Step response comparison of the optimum FOLPD and the original model

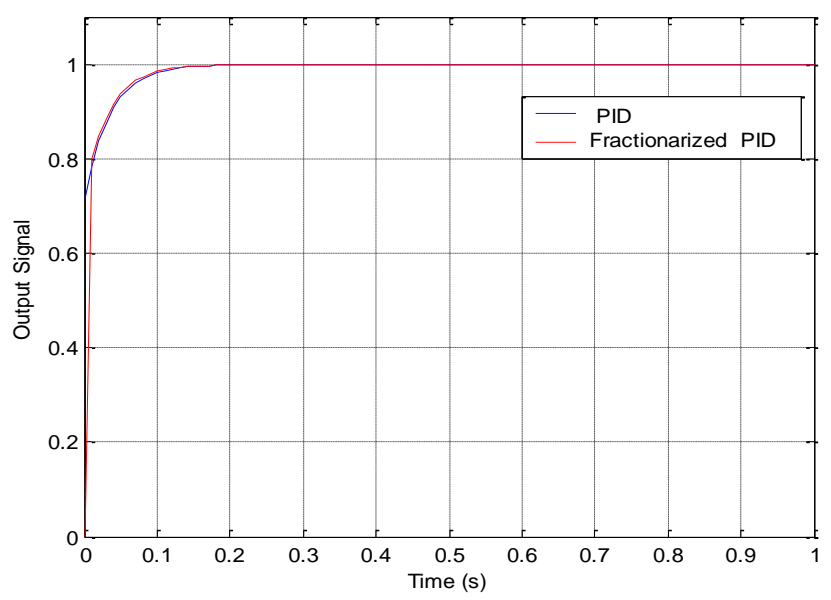

Figure.5 Closed-loop step response of the fractionalized PID and the integer-order PID controller

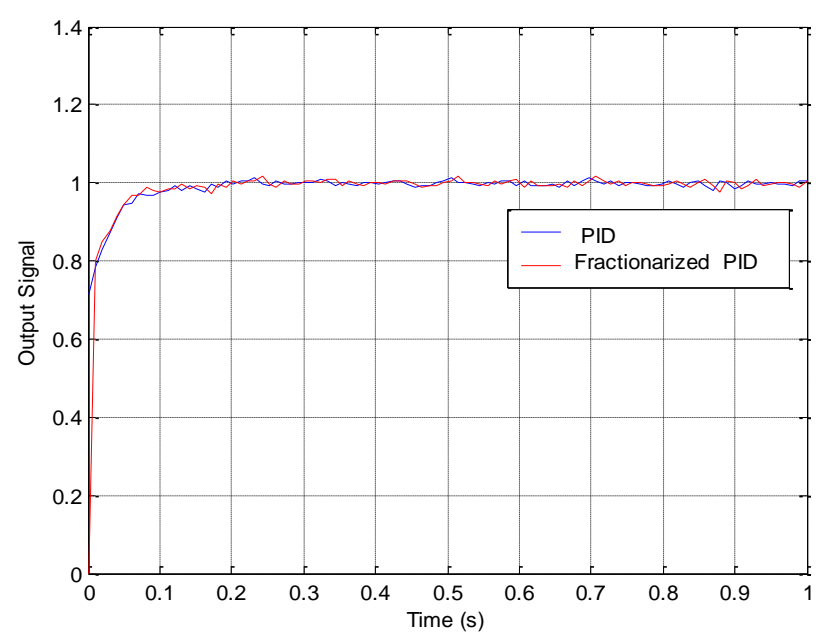

Figure.6 The PID and fractionalized order PID controllers with random output noise of $5 \%$ of the reference signal amplitude $(\alpha=0.4)$

can perform this task and the optimal FOLPD model obtained is given as follows:

$$
G_{r}(S)=\frac{0.9951}{3.5014 S+1} e^{-1.634 S}
$$

The comparison of the open-loop step response is shown in Fig. 4. It can be observed that the approximation is fairly effective.

Designing a suitable feedback controller for the original FO-LTI system $\mathrm{G}$ can be a formidable task. Now let us consider designing an integer-order PID controller for the optimally reduced model $G_{r}(s)$ and let us see if the designed controller still works for the original system.

The integer-order PID controller to be designed is in the following form $[3,4]$ :

$$
C(s)=K_{p}\left(1+\frac{1}{T_{i} s}+\frac{T_{d} s}{\frac{T_{d}}{N} s+1}\right)
$$

The optimum ITAE criterion-based PID tuning formula [4] can be used:

$$
\begin{aligned}
K_{p} & =\frac{\left(0.7303+\frac{0.5307 T}{L}\right)(T+0.5 L)}{K(T+L)} \\
T_{i} & =T+0.5 L \\
T_{d} & =\frac{0.5 L T}{T+0.5 L}
\end{aligned}
$$

The parameters of the PID controller are then $K p=3.4160, T i=3.8164, T d=0.2890$, and the PID controller can be written as

$$
C(s)=\frac{1.086 s^{2}+3.442 s+0.8951}{0.0289 s^{2}+s}
$$

The parmeters of the Fractionalized PID controller are then $\mathrm{Kp}=3.4160, \mathrm{Ti}=3.8164, \mathrm{Td}=0.2890$, $\alpha=0.4$ and the fractionalized PID controller can be written as:

$$
\begin{aligned}
C_{f}(s) & =\frac{1}{s^{\alpha}} \frac{1}{S^{(1-\alpha)}} \frac{\left(1.086 s^{2}+3.442 s+0.8951\right)}{(0.0289 s+1)} \\
& =\frac{1}{s^{0.4}} \frac{1}{s^{0.6}} \frac{\left(1.086 s^{2}+3.442 s+0.8951\right)}{(0.0289 s+1)}
\end{aligned}
$$

Fig. 5 shows the time response characteristics of the PID and fractionalized order PID controllers without Noise. Fig. 6 shows the time response characteristics of The PID and fractionalized order PID controllers with random output noise of $5 \%$ of the reference signal amplitude $(\alpha=0.4)$ and The characteristics are compared in table 1 .

The PID and fractionalized PID controllers with random output noise of $20 \%$ of the reference signal amplitude $(\alpha=0.5)$ is given in the following figure: 




Figure.7 The PID and fractionalized PID controllers with random output noise of $20 \%$ of the reference signal amplitude $(\alpha=0.4)$

\subsection{Robustness analysis}

The evaluation of the control system performance will be realized by dining a quadratic error criterion $\mathrm{J}$ given by,

$$
J_{\alpha}=\int_{t_{I}}^{t_{F}}\left(U_{R}(t)-Y(t)\right)^{2} d t
$$

The Quadratic error criterion with random output noise of $5 \%, 10 \%, 15 \%$ and $20 \%$ are given in Table1, Table 2, Table 3 and Table 4 respectively:

Table 1. Quadratic error criterion with random output noise of $5 \%$

\begin{tabular}{|c|c|c|c|c|c|c|}
\hline$\alpha$ & 0.1 & 0.2 & 0.3 & 0.4 & 0.5 & 1 \\
\hline $\mathrm{J}$ & 0.036 & 0.031 & 0.029 & 0.053 & 0.028 & 0.065 \\
\hline
\end{tabular}

Table 2. Quadratic error criterion with random output noise of $10 \%$

\begin{tabular}{|c|c|c|c|c|c|c|}
\hline$\alpha$ & 0.1 & 0.2 & 0.3 & 0.4 & 0.5 & 1 \\
\hline $\mathrm{J}$ & 0.13 & 0.12 & 0.12 & 0.14 & 0.11 & 0.25 \\
\hline
\end{tabular}

Table 3. Quadratic error criterion with random output noise of $15 \%$

\begin{tabular}{|c|c|c|c|c|c|c|}
\hline$\alpha$ & 0.1 & 0.2 & 0.3 & 0.4 & 0.5 & 1 \\
\hline $\mathrm{J}$ & 0.20 & 0.19 & 0.18 & 0.21 & 0.17 & 0.39 \\
\hline
\end{tabular}

Table 4. Quadratic error criterion with random output noise of $20 \%$

\begin{tabular}{|c|c|c|c|c|c|c|}
\hline A & 0.1 & 0.2 & 0.3 & 0.4 & 0.5 & 1 \\
\hline J & 0.26 & 0.23 & 0.25 & 0.27 & 0.22 & 0.52 \\
\hline
\end{tabular}

Tables 1, 2, 3, and 4 provides the quadratic error criterion with random output noise of $5 \%, 10 \%$, $15 \%$, and $20 \%$ respectively, comparing the fractionalized and the integer responses of PID control, we remark the fractionalized control give them a certain diminution of the noise effect of about 50\% comparatively to the classical PID results.

\subsection{Discussion and remarks}

The proposed fractionalization technique is based on the replacement of rational (integer order) transfer function by a cascaded fractional order elements. The global feedback control system must be equivalent to the original one, for a tolerated approximation error and a working frequency bandwidth. It opens new perspectives for designing more robust and reliable control systems. The following comments can emerge from the examination of the two illustrative applications examples.

The examination of the numerical example results shows that the use of the fractionalization approach is advantageous for disturbance rejection because as it appears in (2), the calculus of the fractional derivative is dependent on all the history of the signal, which moderates the effect of variations and external random noises $[4,6]$.

This robustification leaded to an improvement in noise rejection of about $50 \%$ for PID control.

This new robustification approach can be implemented in a larger class of adaptive and nonadaptive control systems as PID controllers (fractionalization of the integer order integrator).

\section{Conclusion}

In present work, we propose a new approach for PID robust control by introducing fractional order filters in the classical feedback control loop without changing the overall equivalent closed loop transfer function.

The idea was to get benefit from the high performance quality of fractional order systems confirmed in many precedent research works.

The simulations results show the best performances obtained by using the Fractionalized PID comparatively to the classical PID controller and the Fractionalization approach allows improving the noises rejection and the robustness of the control scheme.

Further research will concern the extension of this technique to the fractionalization of more general integer order functions, in order to obtain desired fractional dynamics in the closed loop feedback control system.

\section{References}

[1] Y. Bensafia, S. Ladaci, and K. Khettab, "Using a Fractionalized Integrator for Control 
Performance Enhancement", International Journal of Innovative Computing, Information and Control, Vol. 11, No. 6, pp. 2013-2028, 2015.

[2] Y. Bensafia, S. Ladaci, and K. Khettab, "Using the Sub-optimal Approximation of Fractional Order Transfer Functions to Design Suitable PID Controllers", In: Proc. 13th Int. Conf. on Sciences and Techniques of Automatic Control \& Computer Engineering, Monastir, Tunisia, pp.784-791, 2012.

[3] S. Ladaci and Y. Bensafia, "Fractionalization: A New Tool for Robust Adaptive Control of Noisy Plants", In: Proc. of the 6th Workshop on Fractional Differentiation and Its Applications FDA'2013, part of IFAC Joint conference, Grenoble, France, pp.374-379, 2013.

[4] K. Bettou and A. Charef, "Improvement of Control Performances Using Fractional $\mathrm{PI}^{\lambda} \mathrm{D}^{\mu}$ Controllers", In: Proc. $5^{\text {th }}$ International Symposium on Hydrocarbons \& Chemistry (ISHC5), Sidi-fredj, Algiers, 2010.

[5] F.S. Wang, W.S. Juang, and C.T. Chan, "Optimal tuning of PID controllers for single and cascade control loops", Chemical Engineering Communications, Vol.132, pp.1534, 1995.

[6] A. Djouambi, A. Charef, and A.V. Besançon, "Fractional Order Robust Control Based on Bodes Ideal Transfer Function", RS-JESA, Fractional order systems, Vol.42, pp. 999-1014, 2008.

[7] A. Oustaloup, F. Levron, B. Mathieu, and F. Nanot, "Frequency-Band Complex Noninteger Differentiator: Characterization and Synthesis", IEEE Transactions on Circuits and Systems I, Vol.47, No.1, pp.25-39, 2000.

[8] A. Oustaloup, J. Sabatier, and P. Lanusse, "From fractal robustness to CRONE control", Fractionnal Calculus and applied Analysis, pp. 1-30, 1999.

[9] A. Oustaloup, La Dérivation Non Entière: Théorie, Synthèse et applications, Hermès: Paris, 1995.

[10] Oustaloup and B. Mathieu, La commande CRONE: $d u$ scalaire au multivariable, HERMES, Paris, 1999.

[11] M. Axtell and M.E. Bise, "Fractional calculus applications in control systems", In: Proc. the IEEE National Aerospace and Electronics Conference, New York, USA, pp. 563-566, 1990.

[12] A.J. Calderon, B.M. Vinagre, and V. Feliu, "Fractional order control strategies for power electronic buck converters", Signal Processing, Vol. 86, pp.2803-2819, 2006.

[13] A. Monje, Yang-Quan Chen, Fractional-order Systems and Controls, Fundamentals and Applications, pp.193-194, Springer, 2010.

[14] S. Ladaci, J.J. Loiseau, and A. Charef, "Adaptive Internal Model Control with fractional order Parameter", Int. J. Adaptive Control and Signal Processing, Vol.24, pp.944-960, 2010.

[15] S. Ladaci, A. Charef, and J.J. Loiseau, "Robust fractional adaptive control based on the strictly positive realness condition", Int. J. of Applied Mathematics and Computer Science, Vol.19, No.1, pp. 69-76, 2009.

[16] S. Ladaci and Y. Bensafia, "Indirect fractional order pole assignment based adaptive control", Engineering Science and Technology, an International Journal, Elsevier, Vol. 9, No. 1, pp. 518-530, 2016.

[17] Y. Bensafia, K. Khettab, and S. Ladaci, "DCMotor Velocity Control Using a Robust Fractionalized Adaptive PI Controller', In: Proc. of the 16th International conference on Sciences and Techniques of Automatic control \& computer engineering, STA'2015, Monastir, Tunisia, 2015.

[18] Y. Bensafia and S. Ladaci, "Adaptive control with fractional order reference model", Int. J. of Sciences and Techniques of Automatic control \& computer engineering, IJ-STA, Vol.5, No.2, pp.1614-1623, 2011.

[19] J. Sabatier, A. Oustaloup, AG. Iturricha, and F. Levron, "CRONE control of continuous linear time periodic systems: Application to a testing bench", ISA Transactions, Vol.42, pp.421-436, 2003.

[20] S. Dadras and H.R. Momeni, "Control of a fractional-order economical system", Physica A, Vol.389, pp.2434-2442, 2010.

[21] K. Khettab, Y. Bensafia, and S. Ladaci, "Chattering Elimination in Fuzzy Sliding Mode Control of fractional Chaotic Systems Using a Fractional Adaptive Proportional Integral Controller", International Journal of Intelligent Engineering and Systems, Vol.10, No.5, pp. 255-266, 2017.

[22] Podlubny, "Fractional-order system and fractional-order controllers", Technical report uef-03-94 Institut of Experimental Physics, Academy of Sciences, Slovakia, 1994.

[23] Podlubny, "Fractional Order Systems and $\mathrm{PI}^{\lambda} \mathrm{D}^{\mu}$ Controllers", IEEE Transactions on Automatic Control, Vol.44, No.1, pp.208-214, 1999. 
[24] K. Khettab, S. Ladaci, and Y. Bensafia, "Fuzzy adaptive control of fractional order chaotic systems with unknown control gain sign using a fractional order Nussbaum gain", IEEE/CAA Journal of Automatica Sinica, Vol.4, No.3, pp.1-8, 2017.

[25] K. Khettab, Y. Bensafia, and S. Ladaci, "Fuzzy adaptive control enhancement for non-affine systems with unknown control gain sign", In: Proc. of the 16th Int. IEEE Conf. on Sciences and Techniques of Automatic control and computer engineering, STA'2015, Monastir, Tunisia, pp. 616-621, 2015.

[26] K. Khettab, Y. Bensafia, and S. Ladaci, "Robust Adaptive Fuzzy Control for a Class of Uncertain Nonlinear Fractional Systems", In Recent Advances in Electrical Engineering and Control Applications - Springer International Publishing, pp.276-294, 2017.

[27] K. Khettab, Y. Bensafia, and S. Ladaci, "Robust Adaptive Interval Type-2 Fuzzy Synchronization for a Class of Fractional Order Chaotic Systems", In. Fractional Order Control and Synchronization of Chaotic Systems, Springer-Verlag Germany, pp.203224, 2017.

[28] A. Rajamanickam and S.S. Mohideen, "A Multi-Wavelet Neural Network Particle Swarm Optimization Based MRPID of Interior Permanent Magnet Synchronous Motor Drives", International Journal of Intelligent Engineering and Systems, Vol.9, No.3, pp.53-64, 2016.

[29] A. Bouguerra, D. Saigaa, K. Kara, and S. Zeghlache, "Fault-Tolerant Lyapunov-GainScheduled PID Control of a Quadrotor UAV", International Journal of Intelligent Engineering and Systems, Vol.8, No.2, pp. 1-6, 2015.

[30] M. Salem, M.F. Khelfi, and R.B. Bouiajra, "Statistical Analysis of Harmony Search Algorithms in Tuning PID Controller", International Journal of Intelligent Engineering and Systems, Vol.9, No.4, pp. 98-106, 2016. 